these credentials in the United States at the present time. However, with the recent advent of the CAQ in sports medicine by the American Academy of Family Practice and board certification by the Conjoint Board of Sports Medicine of the American Osteopathic Association, more physicians will be certified. Sports medicine fellowships are also multiplying, with the typical fellowship being of 1year's duration and oriented toward primary care sports medicine.

Physicians-in-training who aspire to careers in primary care sports medicine at the Division I university level would apparently be well advised to complete a residency in family medicine and become board certified, then serve a 1-year primary care fellowship in sports medicine with subsequent sports medicine certification. Orthopedic surgeons who would be team physicians for Division I universities should probably complete a fellowship in orthopedic sports medicine, which is becoming a subspecialty of orthopedics. Because of lack of funds, smaller universities and colleges will probably continue their arrangement of volunteer team physicians (either family physician or orthopedist), but the physician they seek should probably be similarly trained to be competent, to be competitive, and for (the physician's) medicolegal protection.

\section{References}

1. Luckstead EF: Pediatric team physicians. Pediatrics $1986 ; 78(5): 941-942$

2. Kujola UM, Heinonen OJ, Lehto $M$, Järvinen $M$, Bergfeld JA: Equipment, drugs, and problems in competition and team physician. Sports Med October 1988;6(4):198.

3. Tiitinen J, Heikkola R, Hyvärinen $\mathrm{H}$, et al: Experiences from the Medical Service in the Grand Games of Youth. Finnish Sports \& Exercise Medicine 1986;5:98-101.

4. Evarts CM: Sports medicine-the profession/the physician. Am J Sports Med 1990;18(4):438-441.

5. Samples P: The team physician: No official job description. The Physician and Sports Medicine January $1988 ; 16(1): 170-173$.

6. Howe WB: Primary care sports medicine: A parttimer's perspective. The Physician and Sports Medicine January 1988;16(1):103-104.

7. National Association of Collegiate Directors of Athletics: The National Directory of College Athletics. Cleveland, Ohio, Collegiate Directories, Inc, 1996.

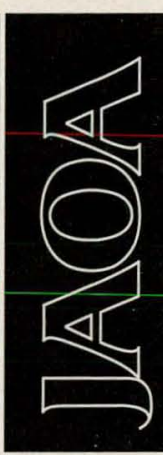

Clinical practice

\title{
Delusional parasitosis: A practical guide for the family practitioner in evaluation and treatment strategies
}

\author{
MICHAEL WINSTEN, DO
}

Delusional parasitosis (DP) is a psychiatric syndrome in which the patient falsely believes he or she is infested with parasites despite clear evidence to the contrary. Patients with DP generally think that mites, lice, or other insects have invaded their skin and most frequently seek treatment from dermatologists or family practitioners. These patients are usually reluctant to seek psychiatric care or to accept a referral to a psychiatrist. This article provides a comprehensive biopsychosocial understanding of the complexities of DP and suggests a practical strategy for evaluation and treatment by the family practitioner. Treatment with antipsychotic drugs, such as pimozide, is discussed as is a treatment team approach involving a psychiatrist, dermatologist, and family practitioner.

(Key words: Parasitosis, delusional; delusional disorder, somatic; folie à deux; psychotic disorder, induced; pimozide; biopsychosocial considerations; treatment; etiology)

$\mathrm{D}$ elusional parasitosis (DP) is a psychiatric syndrome in which patients have a fixed, encapsulated, and false belief that they are infested with parasites. Persons afflicted with this disorder are much more likely to present to dermatologists or family practitioners than to psychiatrists.1,2 This likelihood is problematic because nonpsychiatrists often feel ill-equipped to deal with this type of illness. ${ }^{3}$ The situation is further complicated with the patient's reluctance to accept psychiatric treatment. ${ }^{4} \mathrm{~A}$ number of psychiatric and organic considerations should be considered when treating patients with DP. 5 What follows is a description of a comprehensive biopsychosocial approach to understanding and treating these difficult patients.

Described by Thieberge in 1894 as acaraphobia, $1,2 \mathrm{DP}$ was later mentioned

Dr Winsten is a board-certified psychiatrist and the medical director of the Counseling Center in Alamogordo, New Mexico.

Correspondence to Michael Winsten, DO, The Counseling Center, 1408 8th St, Alamogordo, 88310. by Perrin ${ }^{1}$ as parasitophobic neurodermatitis. More extensive studies $1,2,6-8$ further delineated the multiple, complex characteristics and causes of DP, including a review in Europe by Skott in 1978 that included more than 400 cases.1,2,6 In the 1980 s, Munro ${ }^{8}$ referred to DP as a monosymptomatic hypochondriacal psychosis.

The Diagnostic and Statistical Manual of Mental Disorders, Fourth Edition (DSM-IV) classifies DP as a delusional disorder, somatic type. ${ }^{9}$ Other examples of similarly classified disorders include body dysmorphic disorder, defined as a preoccupation with an imagined defect in appearance that can be held with delusional intensity. ${ }^{9}$ Another is olfactory reference syndrome in which patients believe they are emitting a foul odor. 10-12 In DP and these other syndromes, the psychosis is often limited to one fixed delusion, and the patient may function normally in all other areas of life. If the delusional thought processes are more global, extremely bizarre, or pervasive and mul- 
tifocal, one may conclude that the DP symptoms are a manifestation of a more incapacitating syndrome, such as schizophrenia, obsessive-compulsive disorder, or major depression with psychotic features.13-14

\section{Epidemiologic and clinical characteristics}

Generally heterogeneous, patients with DP come from varied social, economic, and occupational backgrounds. Nonetheless, some characteristics predominate. Older persons are more likely than younger persons to have this disorder. The average age of onset is 51.9 years. Similarly, more women than men are affected, with a 2:1 female-to-male ratio. Female predominance increases with age. 1 In general, patients with DP are resistant to and difficult to treat, leading to frustration for both patient and physician.

There may exist a variety of other possible predisposing factors for developing DP, such as a history of actual infestation with lice or scabies or decreased visual acuity. 3

Approximately $12 \%$ to $25 \%$ of patients present with folie à deux. In other words, a shared delusion is present in which another person (usually the spouse) also mistakenly believes he and his partner are infested. 15

Most frequently, the patient with DP is a middle-aged to elderly woman who is socially isolated. She has a history of "doctor-shopping" with regard to dermatologists or family practitioners. She will often have received multiple, redundant, and sometimes potentially dangerous dermatologic treatments. Evidence of excoriation and skin damage from overuse of "remedies" often exists. This damage can further complicate diagnosis and treatment or worsen symptoms because of secondary irritation.

These patients tend to use bizarre or dangerous home remedies. Consultation of pest control specialists or entomologists commonly occurs. In an attempt to rid themselves of their imagined parasites, patients frequently relocate or repetitively fumigate their home. 16

Sometimes, these patients bring to the office visit small containers filled with mixed debris (excoriated skin particles, hair, paper, lint) to "prove" the parasites exist. This container, known as the matchbox sign, serves only to frustrate physician and patient when no parasites are actually found. 17

Patients with DP often display abnormal personality traits and meet criteria for such personality disorders as schizoid, histrionic, or obsessive-compulsive disorders. 18 The treating physician must realize that DP is a generally well-circumscribed disorder with a separate life of its own enabling the patient to function relatively well in other areas of life. Nonetheless, DP may also be a symptom or manifestation of a more global, pervasive psychiatric depressive disorder or schizophrenia. If this latter scenario is present a different treatment strategy from the one described herein would be used.

\section{Differential diagnosis}

A full organic workup must be completed to evaluate for the source of a patient's abnormal sensations. Obviously, a thorough evaluation by a dermatologist should be conducted to rule out an unusual or insidious dermatologic cause of pruritus or an actual infestation. ${ }^{2}$ Combinations of biologic/organic, psychologic, and social factors may contribute to DP as well. Skott and Lyell emphasize that DP can be traced to multiple causes. ${ }^{5}$ As such, collaboration among professionals (dermatologist, family practitioner, and psychiatrist) proves essential for successful treatment. 19

Substance abuse-particularly cocaine, amphetamine, or chronic alcohol abuse-often coexists as an associated cause of DP. Other drugs, namely corticosteroids and phenelzine, have been linked with DP symptoms.

Parkinson's disease and Huntington's chorea represent two neurologic disorders that should be excluded. So, too, should delusions associated with dementia.

Acquired immunodeficiency syndrome or syphilis or other infectious diseases may be implicated as well. A comprehensive list of conditions associated with DP is provided in the Table.1,2,20
Appropriate laboratory studies should include complete blood cell count, SMA20 blood chemistries, thyroid function tests, measurement of vitamin $\mathrm{B}_{12} /$ folate levels, rapid plasma reagin (test for syphilis), urinalysis, and drug screen. An electrocardiogram (ECG), electroencephalogram, or computed tomography of the brain may also be considered. ${ }^{1}$

Upon interviewing the patient, the physician often can make a psychodynamic formulation that can shed light on the underlying nature and cause of the patient's delusions. 14 For example, the patient may genuinely feel dysesthesia or pruritus but misinterprets this sensation as being caused by parasitic infestation. ${ }^{21}$ Frequently, these sensations represent symbolic misinterpretations based on premorbid psychological or social conflicts, or conflicts involving repressed sexual and/or aggressive desires. This misinterpretation may also be attributable to underlying personality traits or disorders (obsessive/compulsive, hysterical somatization). The delusion accompanying DP may represent a compromise formation where the symptoms stabilize the patient's emotional state. Thus, she may not want to give up this "emotional safety net"; otherwise, she would have to deal with the existence of an emotional problem.

These concepts and the nature of DP perhaps are best illustrated by specific cases. In particular, the case illustrations that follow emphasize the concept of delusions that serve to prevent more severe ego disintegration. They also illustrate repressed conflicts concerning sexuality and aggression. 20,22

\section{Case I}

A 47-year-old white widow presented to an ambulatory care general practice clinic. She was meticulously groomed and dressed. She requested treatment for "tiny mites or bugs burrowing into the back of my neck and upper arms." No physical evidence of infestation was present. Upon further investigation into the patient's history, it was determined that the patient had been seeking treatment for "parasites" at several other clinics, including dermatology and family practice offices. 


\section{Table \\ Conditions Associated With Delusional Parasitosis†}

$\begin{array}{ll}\text { Substance abuse } & \text { Infections } \\ \text { Alcohol } & \text { Tuberculosis } \\ \text { Amphetamines } & \text { AIDS } \\ \text { Cocaine } & \text { Syphilis }\end{array}$

Medications

Phenelzine

Corticosteroids

\section{Endocrine disorders \\ Thyroid disease \\ Diabetes mellitus}

\section{Neurologic origins \\ Dementia \\ Parkinson's disease \\ Huntington's chorea \\ Central nervous \\ system tumors \\ Head trauma}

\section{Psychiatric disorders}

Schizophrenia

Affective disorder

Dementia with delusions

Nondelusional simple phobia

Obsessive/compulsive disorder

\section{Psychodermatologic origins}

Factitial dermatosis

Neurotic excoriation

Münchausen syndrome

Trichotillomania

\section{Malignancies}

Lymphoma

Breast

Chronic lymphocytic

leukemia

†Modified from Johnson and Anton. 2

* Entities specifically causing dysesthesias and pruritus.

The patient indicated that she was exhausted and frustrated that some of the physicians, "didn't even believe that there were any bugs." She stated that several tests and examinations performed by dermatologists were unable to find any bugs because "they are very small and go deep under my skin."

On some occasions, the patient had been treated with topical antiparasitics lindane (Kwell) or permethrin 1\% lice treatment (Nix). While she admitted these treatments provided temporary relief, the "bugs always came back." At least one dermatologist suggested she see a psychiatrist, which caused her to exclaim, "Can you believe the nerve of that doctor?"

Her problem began approximately 2 years earlier after she had had a onetime sexual encounter with a man whom she said "gave the bugs to me." Her husband had died 4 years prior to that aforementioned sexual encounter.

In a supportive fashion, a psychiatry referral was suggested; however, the patient immediately rejected this suggestion and left the office in a hostile manner.

\section{Case 2}

A 74-year-old white woman presented to a dermatologist's office with a long history of reported parasites infesting her torso and upper extremities. She had been to the office on three previous occasions and had been treated with topical antiparasitic agents at her insistence, despite being informed that no evidence was found of infestation.

The patient had multiple excoriations from incessant scratching. She reported bathing in a mixture of water and hydrogen peroxide to "kill the bugs." This treatment only resulted in further skin irritation.

She brought to the office visit a small plastic container of debris, mostly dust, hair, and skin scrapings. She wanted the sample to be examined microscopically to "prove" the bugs existed. (No parasites were found.) The patient previously had refused referral to a psychiatrist.

Her husband accompanied her during this visit; he was equally adamant and frustrated, insisting that his wife was infested and no one had been able to help her. A regimen of low-dose haloperidol (Haldol) was suggested, but she refused this treatment upon being told this drug was "psychiatric" medication.

\section{Case 3}

A 33-year-old white woman reluctantly came to a community mental health center. She had a 1-year history of a persistent belief that insects were infesting her skin and her home. Her family, friends, and physicians had been urging her for months to consider psychiatric treatment. She firmly believed that the bugs were real and felt "ridiculous" in seeking treatment from a psychiatrist.

Preoccupation with bugs had greatly 
affected her daily life and her relationships. She had sent "samples" to entomology laboratories and had her home fumigated regularly to no avail and against her husband's protests. She felt depressed because of her inability to prove or explain the existence of the insects.

She had no history of other delusional themes or psychotic symptoms and had generally functioned well in other areas of her life. Her family practitioner had prescribed sertraline hydrochloride, (Zoloft), $50 \mathrm{mg} / \mathrm{d}$, which was helpful for her mood but did not affect her delusion.

A compassionate, nonconfrontational therapeutic understanding was established, and she agreed to a trial of pimozide (Orap). An initial dosage of $2 \mathrm{mg} / \mathrm{d}$ was titrated to $6 \mathrm{mg} / \mathrm{d}$ over 8 weeks. Visits were scheduled every 1 to 2 weeks to monitor her progress and to evaluate any side effects. Her preoccupation with infestation gradually diminished and was no longer an issue 2 months after initiation of treatment.

\section{Antipsychotic agents}

Several studies during the past 20 years have demonstrated the superior efficacy of the high potency neuroleptic pimozide in treating DP. ${ }^{1}$ Pimozide is an orally active antipsychotic of the diphenylbutylpiperidine class similar to the structure of haloperidol, which is a butyrophenone-class antipsychotic. Two double-blind studies ${ }^{1}$ reported an approximately $90 \%$ response rate to pimozide. In addition, a retrospective study of 60 patients with DP found a $73 \%$ response rate. 3 Pimozide has been used in the United States for treatment of Tourette's syndrome and more extensively in Europe for chronic schizophrenia.

Other high-potency neuroleptics, such as haloperidol and trifluoperazine hydrochloride (Stelazine), may also be effective.11,23 Several explanations have been given regarding the superior efficacy of pimozide for DP. The most commonly proposed mechanism is that unlike other neuroleptic agents, pimozide has opiate antagonist properties that may result in a centrally mediated antipruritic effect. This action is in addition to its antipsychotic properties.

Side effects associated with pimozide mirror those of more commonly used high-potency antipsychotics. ${ }^{1}$ The most significant of these include extrapyramidal reactions, akathisia (motor restlessness), orthostatic hypotension, galactorrhea, sedation, QT prolongation, and tardive dyskinesia. Extrapyramidal reactions may be treated with benztropine mesylate (Cogentin), trihexyphenidyl hydrochloride (Artane), or diphenhydramine hydrochloride (Benadryl). Akathisia may be treated with a reduced dosage of pimozide and/or the addition of low doses of a $\beta$-blocker or benzodiazepine.1,12

The complex biopsychosocial nature of DP necessitates a varied treatment protocol with an emphasis on the following approaches: 3

- Confirm presumptive diagnosis of DP by ruling out actual parasitic infestation, other organic causes (Table), or a more global underlying psychiatric condition.

- Listen to the patient's history in a nonjudgmental, nonthreatening fashion. A therapeutic relationship can be more easily established if the patient feels her doctor is taking her seriously. Nonjudgmental listening is crucial for lessening the patient's frustration and sense of isolation. ${ }^{7}$

- Tactfully ask how the condition has affected her life. Her answer should help the physician judge the severity of the illness and may reveal problem areas that merit further investigation. Be alert to any area where the patient will allow intervention.

- Consider anxiolytic medications to help with reactive symptoms, keeping in mind that such medications will not generally eradicate any delusions. Studies suggest that tricyclic antidepressants, such as nortriptyline hydrochloride (Pamelor) may be helpful, particularly if evidence of underlying depression exists, or if DP may be a psychotic symptom from an otherwise hidden or atypical major depression.10 Hydroxyzine pamoate (Vistaril) may also be beneficial because it has both antipruritic and anxiolytic properties. Another choice may be the antidepressant doxepin hydrochloride with its strong antihistaminic action. 12

- Order appropriate tests before prescribing pimozide, the drug of choice, or any other antipsychotic agent. In addition to a baseline ECG and liver function tests, the patient's serum electrolyte count and levels of calcium, phosphorous, magnesium, and blood urea nitrogen/creatinine should be ascertained.1,3 Cardiac status via ECG should be monitored periodically during treatment with pimozide.

Contraindications to pimozide treatment include congenital long QT syndrome, a significant history of cardiac arrythmia, concomitant use of drugs that increase the QT interval, and previous known allergy or severe adverse reactions to pimozide.

Initiate treatment at $1 \mathrm{mg} / \mathrm{d}$, increasing by $1 \mathrm{mg}$ every 5 to 7 days based on the patient's response. Single or divided doses may be prescribed. Many patients show a response at $4 \mathrm{mg} / \mathrm{d}$ or less. To minimize the possibility of adverse effects and to lessen the likelihood of tardive dyskinesia, it is best to maintain a dosage of less than $10 \mathrm{mg} / \mathrm{d}$, with an absolute maximum of $20 \mathrm{mg} / \mathrm{d} .{ }^{1}$ It may take several weeks before a response is noted. Nonetheless, the physician should attempt to find the minimum effective dosage for maintenance therapy. The dosage may be gradually tapered or discontinued after several months, but many patients relapse after discontinuation of the drug, thereby necessitating reinstitution of pimozide. ${ }^{1}$

\section{Further considerations}

A great deal of controversy can be found in the literature regarding treatment of DP by nonpsychiatrists. 1,7 Ultimately, the physician's comfort level should be weighed against the risks/benefits of treatment and/or the patient's potential for continued suffering without treatment. Repeated attempts by the family practitioner to forge an alliance with a psychiatrist are recommended but not always possible. 
Informed consent may also be an issue. A patient who adamantly refuses any psychiatric explanation for her symptoms is likely to refuse treatment with neuroleptics if she is told the drug is a psychiatric medication. The physician may wish to present the pimozide as a means to "help with the uncomfortable itching feeling" or to "keep the bugs from bothering you." The physician may choose to describe potential side effects without specifically referring to its psychiatric application. Again, such an omission poses an ethical dilemma; whether the physician chooses to take this approach depends on the patient's severity of illness, risks/benefits of treatment, and the physician's comfort level and philosophy.

Another consideration for improving the treatment of patients with DP is the establishment of Psychiatry/Dermatology liaison clinical training during residency. Such a liaison would establish a forum for the exchange of insights and information about such disorders in which effective treatment demands the coordination of two different medical specialties.

Such an effort has been undertaken at Stanford University by Gould and Gragg. ${ }^{24}$ Patients with DP and other psychocutaneous disorders are evaluated by dermatology and psychiatry residents together. A comprehensive treatment regimen is then prescribed. If this coordinated practice can be instituted on a wider basis, it is more likely that the treatment outcomes of such complex patients will significantly improve.

\section{References}

1. Driscoll MS, Rothe MJ, Grant-Kels JM, Hale MS: Delusional parasitosis: A dermatologic, psychiatric, and pharmacologic approach. JAm Acad Dermatol 1993;29:1023-1033.

2. Johnson GC, Anton RF: Delusions of parasitosis: Differential diagnosis and treatment. South Med J 1985;78:914-918.
3. Bond WS: Delusions of Parasitosis: A case report and management guidelines. Annals of Pharmacotherapy 1989;23:304-306.

4. Mitchell C: Successful treatment of chronic delusional parasitosis. Br J Psychiatry 1989; 155:556-557.

5. Berrios GE: Delusional parasitosis and physical disease. Compr Psychiatry 1985;26:395403.

6. Reilly TM: Delusional infestation. Br J Psychiatry 1988;153:44-46.

7. Gould WM, Gragg M: Delusions of parasitosis: An approach to the problem. Arch Dermatol 1976;112:1745-1748.

8. Munro A: Monosymptomatic hypochondriacal psychosis. Br J Psychiatry 1988;153:37-40.

9. Diagnostic and Statistical Manual of Mental Disorders, ed 4. Washington, DC, American Psychiatry Association, 1994.

10. Brotman AW, Jenike MA: Monosymptomatic hypochondriasis treated with tricyclic antidepressants. Am J Psychiatry 1984;141:16081609.

11. Andrews E, Bellard J, Walter-Ryan W: Monosymptomatic hypochondriacal psychosis manifesting as delusions of infestation: Case studies of treatment with haloperidol. J Clin Psychiatry 1986;47:188-190.

12. Koo JYM, Pham CT: Psychodermatology: Practical guidelines on pharmacotherapy. Arch Dermatol 1992;128:381-388.

13. Musalek M, Bach PV, Jaeger S: The position of delusional parasitosis in psychiatric nosology and classification. Psychopathology 1990; 23:115-124.

14. Lyell A: Delusions of parasitosis. Br J Dermatol 1983;108:485-499.
15. Macaskill ND: Delusional parasitosis: Successful nonpharmacological treatment of folie à deux. Br J Psychiatry 1987;150:261-263.

16. Fischer M, Orengo I, Rosen T: Recognizing and helping patients who harm themselves. Consultant 1991;31:21-31.

17. Munro Al, Chmara J: Monosymptomatic hypochondriacal psychosis: A diagnostic checklist based on 50 cases of the disorder. Can J Psychiatry 1982;27:374-376.

18. Reilly TM, Batchelor DH: The presentation and treatment of delusional parasitosis: A dermatological perspective. International Clinical Psychopharmocology 1986;1:340-353.

19. Osman AA: Monosymptomatic hypochondriacal psychosis in developing countries. $\mathrm{Br} J$ Psychiatry 1991;159;428-431.

20. May WW, Terrenning MS: Case reports: Delusional parasitosis in geriatric patients. Psychosomatics 1991;32:88-93.

21. Berrios GE: Tactile hallucinations: Conceptual and historical aspects. Journal of Neurology, Neurosurgery, Psychiatry 1982;45:285-293.

22. Paulson MJ, Petrus EP: Delusions of parasitosis: A psychological study. Psychosomatics 1969;10:111-120.

23. King BH: Hypothesis: Involvement of the serontonergic system in the clinical expression of monosymptomatic hypochondriasis. Pharmacopsychiatry 1990;23:85-89.

24. Gould WM, Gragg TM: A dermatology-psychiatry liaison clinic. J Am Acad Dermatol 1983; 9:73-77. 